\title{
EXPERIMENTAL CHARACTERIZATION AND MITIGATION OF SPECIMEN CHARGING ON THIN FILMS WITH ONE CONDUCTING LAYER
}

\author{
Kenneth H. Downing ${ }^{1}$, M. R. McCartney ${ }^{2}$, and Robert M. Glaeser ${ }^{1,3}$
}

keywords: specimen charging, thin films, cryomicroscopy, electron crystallography

${ }^{1}$ Life Sciences Division, Lawrence Berkeley National Laboratory, Berkeley, California 94720-0001

${ }^{2}$ Center for Solid State Science, Arizona State University, Tempe, Arizona 85287-1704

${ }^{3}$ Department of Molecular and Cell Biology, Stanley/Donner ASU, University of California, Berkeley, California 94720-3206 


\begin{abstract}
Specimen charging may be one of the most significant factors that contribute to the high variability generally low quality of images in cryo-electron microscopy. Understanding the nature of specimen charging can help in devising methods to reduce or even avoid its effects and thus improve the rate of data collection as well as the quality of the data. We describe a series of experiments that help to characterize the charging phenomenon which has been termed the Berriman effect. The pattern of buildup and disappearance of the charge pattern have led to several suggestions for how to alleviate the effect. Experiments are described that demonstrate the feasible of such charge mitigation.
\end{abstract}

\title{
INTRODUCTION
}

Specimen charging occurs when nonconducting specimens are examined in the transmission electron microscope, since inelastic scattering events lead to ionization and the consequent emission of secondary electrons. The preparation of samples on an electrically conducting support film (e.g. carbon film), or subsequent coating of samples with such films, can effectively eliminate many of the well-known symptoms of specimen charging. However, as is reviewed in the preceding paper (Glaeser and Downing, submitted) a more subtle manifestation of specimen charging can still remain in such specimens. As has been noted by Dr. John Berriman, a previously illuminated area generates a phase contrast image in which the irradiated area has a greater inner potential relative to its surround. This effect demonstrates that local charging still occurs in spite of the presence of a conducting layer as a part of the structure of the specimen (Brink et al., 1998b). 
Although the accumulation of positive charges within the nonconducting layer(s) of the specimen must be compensated by matching negative charges within the conducting film, it is possible that both mechanical and electron optical disturbances can still occur due to the resulting capacitative charging (Glaeser and Downing, submitted). Experimental procedures that are able to reduce the buildup of specimen charging might thus lead to significant improvement in image quality, especially at high resolution and with highly tilted specimens. We have therefore begun to investigate whether specimen charging, as detected by its associated increase in phase contrast, can be held to reduced levels by further intervention in the charging process.

Two forms of specimen charging have been observed in the course of these experiments. The first, which we will now refer to as Type I charging, is already discussed commonly among those who are familiar with Berriman's charging-related contrast effect. Type I charging is characterized by the fact that it is readily discharged when the electron beam is moved to a second position, but it remains indefinitely if the electron beam is simply turned off. The second form of specimen charging, which we now refer to as Type II charging, is not discharged when the electron beam is moved to a new position. While both forms of charging reach a limiting (saturated) level, Type II charging (when it occurs) is also observed to build up more slowly than Type I charging, and to reach a markedly higher level of contrast.

The systematic investigation of specimen charging has been made difficult by the poor level of reproducibility of the effect from one specimen to the next. The work reported here has therefore used artificial specimens in which silicon monoxide has been evaporated onto one or both surfaces of a carbon film, as well as conventional biological samples. The improved experimental consistency that is obtained with the artificial specimens gives us greater confidence that others can reproduce the results that we describe here without difficulty. We caution, however, that these artificial specimens 
may not capture all of the effects that might occur in real, biological samples. Conversely, the SiOcoated carbon films may well exhibit charging behaviors that do not occur in the typical, biological specimens. Nevertheless, the use of a defined specimen with reproducible behavior seems to be a necessity at this early stage of investigation.

The results obtained so far have shown that both conventional and unconventional approaches to reducing specimen charging have, at best, inconsistent effects when an electrically conducting film (carbon film, in this case) is included as one component of the specimen. Insertion of an objective aperture for example, which markedly reduces specimen charging on self-supported insulating films, has no significant effect on the phase-contrast effect reported by Berriman. Examination at high ambient gas pressure, which has proven to be very effective in reducing specimen charging in the "environmental scanning electron microscope", does not appear to be a promising approach for transmission EM samples. The use of conductive coatings is seen to eliminate the effect sometimes, but not always (Brink et al., 1998a). The use of conductive coatings nevertheless remains a promising approach if their contrast does not mask the particles of interest. Finally, we show here that Type I charging can be limited to very low levels by repeatedly switching the electron beam to an area different from that which is used to record an image. Unfortunately, flickering the illumination onto and away from the area being imaged would not limit the buildup of Type II charging, and no remedy for that type of specimen charging can be suggested at this time.

\section{MATERIALS AND METHODS}

$\underline{\text { Sample preparation }}$ 
Carbon films with a thickness of 10 to $15 \mathrm{~nm}$ were evaporated onto cleaved mica and floated onto deionized water. These films were then picked up on copper grids. Powdered silicon monoxide (Balzers AG, Liechtenstein) was evaporated from a molybdenum boat at a vacuum initially better than $10^{-5}$ Torr; interestingly, the vacuum improved by a factor of 2 to 3 during evaporation, the condensing film apparently serving as an effective getter. The thickness of the evaporated SiO film was controlled to be about 100 to $150 \mathrm{~Hz}$, as measured with a quartz film-thickness monitor which is calibrated to $10 \mathrm{~Hz} / \mathrm{nm}$ film thickness.

Glucose-embedded purple membrane samples were prepared by allowing a droplet of the membrane suspension to sit on the carbon film for about $30 \mathrm{sec}$, then rinsing with $0.5 \%$ glucose and blotting the grid.

\section{Observation of specimen charging}

Two, quite different methods were used to observe the positive phase-contrast "footprint" that is left on a previously illuminated area of the specimen. In the majority of experiments performed at Berkeley, computer control of the beam-deflection coils, lens currents and a beam shutter was used to illuminate one or more spots in series and then to observe the result with a larger field of illumination. At other locations the illuminated area was kept constant, and the contrast generated at the edge of the footprint could be seen briefly, immediately after the sample was translated. In this second method the sample needs to be moved only enough to bring a region of new, previously unirradiated sample into the electron beam, but not so much as to move all of the previously illuminated area out of the beam. 
In order to easily see and record the footprint due to specimen charging, highly defocused images were produced by operating the electron microscope in diffraction mode, with a relatively high camera length (e.g. 4 meters at $300 \mathrm{kV}$ or 6 meters at $120 \mathrm{kV}$ ). The diffraction pattern was then overfocused (the lens current was adjusted to be higher than that which would properly focus the unscattered beam). The phase-contrast footprint is more easily seen if the amount of overfocus is not too large, and as a result the intensity at the center of the defocused, unscattered beam is noticeably higher than at the perimeter of the defocused image. The electron beam intensities used were varied over the range of 1 electron $/ \mathrm{nm}^{2}$-s to 10 electrons $/ \mathrm{nm}^{2}$-s; these intensities correspond to photographic exposure times of 100 s to 10 s at 10,000 magnification, respectively, as indicated by the automatic exposure meter of the electron microscope.

\section{RESULTS AND DISCUSSION}

$\underline{\text { Two types of charging behavior are observed on SiO-coated carbon films }}$

As was already mentioned in the Introduction, specimen charging (as manifested by the phase-contrast footprint) is often discharged by irradiating an adjacent area, and we designate this as Type I charging. Figure 1 illustrates the nature of this phenomenon. A series of 16 spots, each about $1 \mu \mathrm{m}$ in diameter in a $4 \times 4$ array, was exposed to $\sim 500 \mathrm{e} / \mathrm{nm}^{2}$. The beam was then spread to about 10 $\mu \mathrm{m}$ diameter, and an image was recorded before the pattern fades, which takes just a few seconds. A dark disk is seen corresponding to each of the exposed areas. In fig. 1a, the first spots in the series have faded significantly, leaving only the last with maximum contrast. We interpret this as an 
indication that subsequent exposure can remove the previously built-up charge in an adjacent area. With increasing spot separation, though, there is less of a discharging effect, as is seen in fig. $1 \mathrm{~b}$.

With longer exposures, but not in all specimens, the amount of specimen charging may increase to ultimately give significantly greater phase-contrast which, in addition, is no longer decreased by irradiating adjacent areas of the specimen. In this case one can see a series of overlapping phase-contrast footprints, such as is shown in fig. 2. Images of this type demonstrate that Type II contrast reflects a saturable charging effect, as does Type I contrast. There are, for example, no contrast steps in overlapping footprints as there would be if the footprints were caused by the buildup of hydrocarbon contamination.

Type I and Type II charging effects occur equally well for samples coated with $\mathrm{SiO}$ on either one or both sides of a carbon film. Furthermore, the same phase-contrast effect is observed both when the $\mathrm{SiO}$ layer faces toward the objective lens and when it is facing away from the objective lens. These observations seem to rule out the electrostatic potential of the dipole sheet as being the source of contrast. It therefore seems likely that the positive phase-contrast effect reflects a small amount of uncompensated positive charge that remains distributed over the irradiated area, in agreement with the physical argument discussed in the preceding paper (Glaeser and Downing, submitted).

A useful indication of the range of the discharging seen with the type-I effect can be obtained from observation of the disappearance of a widely distributed charge pattern, such as seen in fig. 3 . Charge footprints were produced either by repeatedly scanning the beam through a row of small spots or simply illuminating a large area. A small beam was then positioned near the previously exposed area for a sufficient time to discharge some of the initial pattern. While the effective range of 
discharging is likely to vary from one material to another, it is clear that there is a characteristic distance of just a few micrometers in this process.

Measurement of the potential by electron holography

Electron holography provides a means to measure the potential distribution on the specimen directly. Normally, the exposure used to record a hologram is several orders of magnitude higher than that which causes complete disappearance of the charge pattern. However, we were able to adapt low-dose techniques with the biprism holography setup in the Center for Solid State Science at Arizona State University to record holograms that provide a useful estimate of the potential associated with the Berriman effect. Figure 4 illustrates the experiment and typical results. The holograms were recorded with an exposure of $\sim 0.015 \mathrm{e} / \mathrm{nm}^{2}$ and are thus fairly noisy. However, we can clearly interpret the variation in phase to indicate a potential difference of $20-30$ volts between the illuminated and unilluminated areas. The maximum slope of the phase indicates an electric field of approximately $18 \mathrm{MV} / \mathrm{cm}$, well within the range of electrical breakdown voltages of insulating materials. Thus it appears, as expected, that the maximum charge buildup is limited by the dielectric strength of the insulating component of the film.

The objective aperture has no subjective effect

While it is generally agreed that specimen charging is a significant concern in electron cryomicroscopy of biological specimens, it is also generally thought that charging can be reduced or even prevented by using a clean objective aperture when recording high-resolution images. There 
can be no argument that the use of an objective aperture greatly reduces the net charge that builds up on self-supported, insulating specimens. Presumably, secondary electrons produced by impact of high energy electrons scattered by the specimen are captured by the positive charge that builds up on the sample. Our observation of the Berriman effect, however, indicates that insertion of an objective aperture has no significant influence either on the amount of charging or on the kinetics of the charging effect when the trapped, positive charges are already "compensated" by the capacitative charge that can flow within a conducting layer of the specimen.

Figure 5 shows a set of representative images which document the fact that there is no subjective difference in the charging effect that is observed with an objective aperture (panels (a) to (e), respectively) or without an aperture (panels (f) to (j), respectively). An area of the sample was exposed to build up the charge, and then a series of images was recorded as the pattern faded with subsequent exposure. There was no detectable difference in either the maximum charge or decay rate whether the objective aperture was in or out.

Any number of schemes could be contrived to increase the number of scattered electrons that hit the objective aperture or other areas near the specimen, thereby generating secondary electrons with the intent of neutralizing positive charges on the specimen. Examples of schemes that we have considered include repeated deflection of the beam onto the objective aperture as part of a "flicker illumination" scheme, and the use of strongly scattering, microfabricated support films to increase the fraction of electrons that are scattered onto the objective aperture. It now seems unlikely that any of these techniques would lead to reduction of this particular charging phenomenon.

The absence of an objective-aperture effect for specimens that already include a conducting layer suggests that low-energy secondary electrons produced in the environment surrounding the specimen have little role, if any, in limiting the amount of charging, i.e. in reaching a saturating level 
of phase contrast. Since the net charge on the sample will, of course, always be zero when there is a conducting layer, it may be the case that the electrostatic potential caused by formation of a dipole sheet is very short-ranged compared to the Coulombic potential that would result from a sheet of equivalent charge. Thus the electric field distribution that gives rise to the Berriman effect may be too weak to capture low-voltage secondary electrons produced at the objective aperture, and apparently even those produced when adjacent areas of the specimen are illuminated.

The latter remark, although only a conjecture, nevertheless raises the point that bulk conductivity induced in the insulating specimen material during irradiation may represent the primary factor limiting the buildup of positive charge. While most of the literature concerning beam-induced conductivity relates to electrons that flow within the illuminated area, there is ample evidence that the velocities and lifetimes of electrons excited into conduction bands of an insulator are sufficient to affect the charge distribution several micrometers from the edge of the beam (Ehrenberg and Gibbons, 1981). Line profiles of conductivity induced by irradiation near grain boundaries demonstrate directly that effects can propagate several micrometers from the beam (Holt, 2000). This interpretation is further supported by the observation that the charge effect is significantly more prominent when the specimen is cooled than when at room temperature, as the beam-induced conductivity is generally weaker for amorphous materials at low temperatures. (Ehrenberg and Gibbons, 1981).

$\underline{\text { High ambient gas pressure has little effect }}$

Specimen charging has been observed to be markedly reduced in the environmental scanning electron microscope, which can operate with the specimen in a gas at relatively high pressure (Toth et al., 2002). Differentially pumped specimen stages have been developed which make it possible to 
study specimens by transmission electron microscopy at gas pressures of a few millibar or more. Experiments have been carried out with facilities of this type located both at ASU and at Haldor Topsøe A/S in Denmark. In both cases images were recorded on TV cameras mounted at the end of a Gatan image filter, which represented the standard mode of operation on these instruments. Both instruments were also operated at $300 \mathrm{kV}$, and the filter was set to transmit the no-loss electrons.

No effect on the charging phenomenon was observed in either microscope. The Copenhagen instrument could be operated at the highest pressures, up to $15 \times 10^{-3}$ bar. At this pressure, the image intensity is substantially less than that seen under normal vacuum conditions (about $2 \times 10^{-5}$ bar) due to scattering by gas introduced into the environmental cell, but the rate of charging, maximum contrast, and rate of discharge are not measurably different from those at high vacuum. There is no practical reason to investigate higher gas pressures, since the further loss of electrons due to scattering by the gas would soon become a severe disadvantage.

Coating biological samples with conducting films can prevent specimen charging

Coating samples with a conductive layer has previously been shown to eliminate the most severe charging problems seen with insulating specimens. Carbon coating is generally used to avoid charging with plastic sections and has been shown to be effective with ice-embedded samples as well (Jakubowski et al., 1989). Sputter coating with materials such as chromium is preferred in SEM work, since very thin and nearly amorphous films can be obtained. However, we and others (Brink et al., 1998a) have found that sputtering causes serious damage to biological samples, as evidenced by the loss of high resolution diffraction from coated protein crystals. This effect is presumably a result of the high energy of sputtered atoms impacting on the sample (Tolmachev, 1994). Thermal evaporation of conductive layers has thus been used in our tests. 
In an effort to obtain films with a higher conductivity than carbon and also to avoid the tremendous increase in resistivity of carbon at low temperatures (Rader and Lamvik, 1992), we have tested platinum-carbon films evaporated from an electron gun onto glucose-embedded purple membrane samples. As shown in fig. 6a, uncoated glucose-embedded samples generally exhibit a prominent Berriman effect. Platinum-carbon coating can reduce the effect to an undetectable level, as shown in fig. 6c. However, for reasons that are not yet clear, the effect is not as reproducible as one would need for routine use. Even on the same grid, the degree of charge reduction provided by the coating can vary from one area to another. There is one simple explanation that could account for such variation: it is clear that the coating must be applied to the glucose-side of the grid; applied to the back side, there is no apparent protection from the Berriman effect. While we believe that all of the purple membranes are on the same side of the grid, we can not exclude the possibility that some of the membranes, as well as some glucose, may have ended up on the side of the grid that was not platinum-carbon coated, accounting for the variability of the coating effect.

The granularity introduced by a PtC film is not a problem with crystalline specimens such as purple membrane, but could pose a limiting difficulty for single particle work where one must be able to identify low contrast objects in a noisy background. Development of more highly amorphous coatings will be a benefit for these specimens.

Condensation of water vapor at low temperature is sufficient to cause specimen charging.

An important issue in cryo-electron microscopy is whether we can hope to completely eliminate charging problems by coating the sample with a conductive layer. The advantage of coating the non-conductive part of the specimen would be lost if further non-conductive material 
were to condense on the sample. Condensation of water is a potential problem when the grid is cooled to below about $-130 \mathrm{C}$. Although a number of other gasses would be able to condense at liquid helium temperatures as well, we have found that water is the primary contaminant in the JEOL-3000-SFF with the specimen at $4 \mathrm{~K}$, and does indeed generate a Berriman-type effect. Figure 7 illustrates the somewhat different nature of the effect seen at low temperature. A carbon film that initially showed no Berriman effect, even at $4 \mathrm{~K}$, was left in the microscope long enough to have accumulated a significant layer of condensed water. As before, an area about $1 \mu \mathrm{m}$ in diameter was illuminated and the beam expanded. In this case, a ring appears around the illuminated area rather than a disk, and streaks are seen radiating away from the area, suggestive of a mechanism of limiting the charge related to electrical breakdown in the material.

\section{Flicker-illumination is one remaining option for mitigating Type I charging}

We had proposed some time ago that specimen charging and its subsequent effects could be limited by recording the image using what we termed "flicker spot" illumination. The beam would be alternately positioned on the area of interest and on an adjacent area in a series of recording and discharging steps. Each incremental exposure would thus alternate with periods where the charge is reduced by exposure of the adjacent area. The recorded image would then be built up with a number of partial exposures, within which the charge buildup is limited to a small fraction of the maximum value. This scheme is somewhat complicated by the fact that in order to fully discharge an area takes a substantially larger exposure than is required to build up the charge, and that this full "discharge exposure" would be required after each partial recording exposure in order to bring the charge back effectively to zero. In order to keep the total time needed to record an image to an acceptably short 
period, it is therefore necessary to increase the beam intensity by a large factor every time that it is shifted to the off-center position.

Nevertheless, we have demonstrated that this procedure can reduce the charge buildup to a very small level. Figure 8 illustrates the effect and some of the required operational parameters. Figure 8a shows the charge pattern built up in an area about $1 \mu \mathrm{m}$ in diameter with an exposure of $\sim 1000 \mathrm{e} / \mathrm{nm}^{2}$. In figs. $8 \mathrm{~b}, \mathrm{c}$ and $\mathrm{d}$, the same area is shown after the same exposure but employing the flicker spot technique to limit the charge buildup as described in the caption.

\section{CONCLUSION}

The Berriman effect is a charge pattern that can often be seen following irradiation of a specimen by an exposure comparable to what is used in cryo-electron microscopy of biological materials. The buildup and stochastic nature of this charge during a normal exposure may play a significant role in the observation that the average quality of cryo-EM images is well below what would be found in an ideal image. We have characterized the appearance of the charge pattern as well as conditions under which it can be erased by subsequent exposure. The results of these experiments suggested several ways in which the charge buildup could be minimized. Applying a conductive coating to the insulating side of the sample appears to be a promising approach, but is not yet routinely effective. Production of abundant secondary electrons in the vicinity of the sample, either through the use of an objective aperture or an environmental cell with high gas pressure, is not found to have a significant influence. "Flicker spot" illumination, in which the beam alternately illuminates the area of interest and an adjacent area, could be effective if the adjacent exposure can be made at an intensity several orders of magnitude higher than on the recorded area so that the total exposure time remains within reason. 


\section{ACKNOWLEDGEMENTS}

This work has been supported in part by National Institutes of Health Grant GM51487 (KHD, RMG), and by the Office of Health and Environmental Research, U. S. Department of Energy under Contract DE-AC03-76F00098. We acknowledge use of facilities in the Center for High Resolution Electron Microscopy at Arizona State University and thank Renu Sharma at ASU and Poul Hansen at Haldor Topsøe A/S, Lyngby, Denmark for help with the environmental cell experiments..

\section{FIGURE LEGENDS}

Figure 1. Type I charging and discharging. A set of 16 exposures was made in a two-dimensional raster, starting at the lower left. Fading of the pattern with subsequent exposures is more prominent with closely spaced spots.

Figure 2. Type II charging. The charge pattern is seen after four overlapping exposures.

Figure 3. Range of discharging. The left column shows three patterns of charge buildup, and the right column shows the pattern after the same exposure followed by exposure of a single spot for 10 sec. In (a) and (c), exposures were with a $0.8 \mu \mathrm{m}$ diameter spot that was scanned 30 times along the row to give a total exposure of about $50 \mathrm{e} / \mathrm{nm}^{2}$ in each spot. The spot was then positioned near the 
end (b) or middle (d) of the row, as indicated by the dark spot that is not present in (a) or (c), for an exposure of about $300 \mathrm{e} / \mathrm{nm}^{2}$. In (e), the initial exposure was about $100 \mathrm{e} / \mathrm{nm}^{2}$ in a $10 \mu \mathrm{m}$ diameter spot, followed by an exposure of about $300 \mathrm{e} / \mathrm{nm}^{2}$ in an $0.8 \mu \mathrm{m}$ diameter spot at the edge of the larger area (f). In each case, subsequent exposure causes disappearance of the charge pattern within a few micrometers of the illumination

Figure 4. Holographic determination of potential in charged region. An area at the edge of the carbon/SiO film was illuminated as indicated in the schematic at left. The beam was then shifted so that the interference pattern, generated by the biprism, moved onto the area of interest, and the hologram was recorded. Image at right represents the phase, which is seen to change by about 40 radians from upper left to lower right across the edge of the previously illuminated area. This phase shift corresponds to a potential difference of around $25 \mathrm{~V}$. (Field of view of the phase image is $7.56 \mu \mathrm{m}$.

Figure 5. Lack of effect of objective aperture on charge phenomenon. The area at the center of the image, about $1 \mu \mathrm{m}$ in diameter, was exposed to $\sim 50 \mathrm{e} / \mathrm{nm}^{2}$. The first image in each series was then recorded with the beam spread. The rate of discharge was then monitored in a series of exposures by focusing the beam back to the original size but shifted to the area just off the image for 1 sec before being spread to record the next image in the series.

Figure 6. Effect of a platinum-carbon film. Half of one grid containing glucose-embedded purple membrane was coated with about $80 \mathrm{~Hz}$ of PtC, as measured on a Balzers QSG-201 thin film thickness monitor. The uncoated area shows a distinctive Berriman effect, seen in (a) as a darker disk 
within one of the membranes, which disappears upon further illumination (b). A coated area (c, d) shows no Berriman effect when examined under the same conditions.

Figure 7. Charge effect on condensed water at low temperature. The prominent light disk to the lower right of the center is an area that was exposed to the beam long enough to remove the condensed water. The fainter area at the center was then exposed to $\sim 1000 \mathrm{e} / \mathrm{nm}^{2}$ before this image was recorded.

Figure 8. Control of charging with flicker-spot illumination. (a) Charge pattern induced by a single exposure of $\sim 1000 \mathrm{e} / \mathrm{nm}^{2}$ in $0.28 \mathrm{sec}$. (b) Same total exposure given to the same area, but fractionated into ten $0.028 \mathrm{sec}$ increments alternating with exposure of the area at the top of the image to $\sim 100000 \mathrm{e} / \mathrm{nm}^{2}$ in $2 \mathrm{sec}$. (c) Exposure fractionated to twenty $0.014 \mathrm{sec}$ increments, alternating with $\sim 100000 \mathrm{e} / \mathrm{nm}^{2}$ to the adjacent area after each incremental exposure. (d) Same exposure to the center area as in c, but adjacent area exposed to $\sim 200000 \mathrm{e} / \mathrm{nm}^{2}$. 


\section{REFERENCES}

Brink, J., Gross, H., Tittmann, P., Sherman, M.B. and Chiu, W. (1998a) Reduction of charging in protein electron cryomicroscopy. J. Microsc., 191, 67-73.

Brink, J., Sherman, M., Berriman, J. and Chiu, W. (1998b) Evaluation of charging on macromolecules in electron cryomicroscopy. Ultramicroscopy, 72, 41-52.

Ehrenberg, W. and Gibbons, D. (1981) Electron Bombardment Induced Conductivity. Academic Press, New York.

Glaeser, R. and Downing, K. (submitted) Specimen charging on thin films With one conducting layer: Discussion of physical principles. .

Holt, D.B. (2000) The remote electron beam-induced current analysis of grain boundaries in semiconducting and semi-insulating materials. Scanning, 22, 28-51.

Jakubowski, U., Baumeister, W. and Glaeser, R.M. (1989) Evaporated carbon stabilizes thin, frozenhydrated specimens. Ultramicroscopy, 31, 351-356.

Rader, R.S. and Lamvik, M.K. (1992) High-conductivity amorphous TiSi substrates for lowtemperature electron microscopy. J. Microsc., 168, 71-77.

Tolmachev, A.I. (1994) On the energy distribution of sputtered atoms at normal ion incidence. Nucl. Instrum. Meth. Phys. Res. B, 93, 415-420.

Toth, M., Phillips, M., Craven, J., Thiel, B. and Donald, A. (2002) Electric fields produced by electron irradiation of insulators in a low vacuum environment. J. Appl. Phys., 91, 4492-4499. 

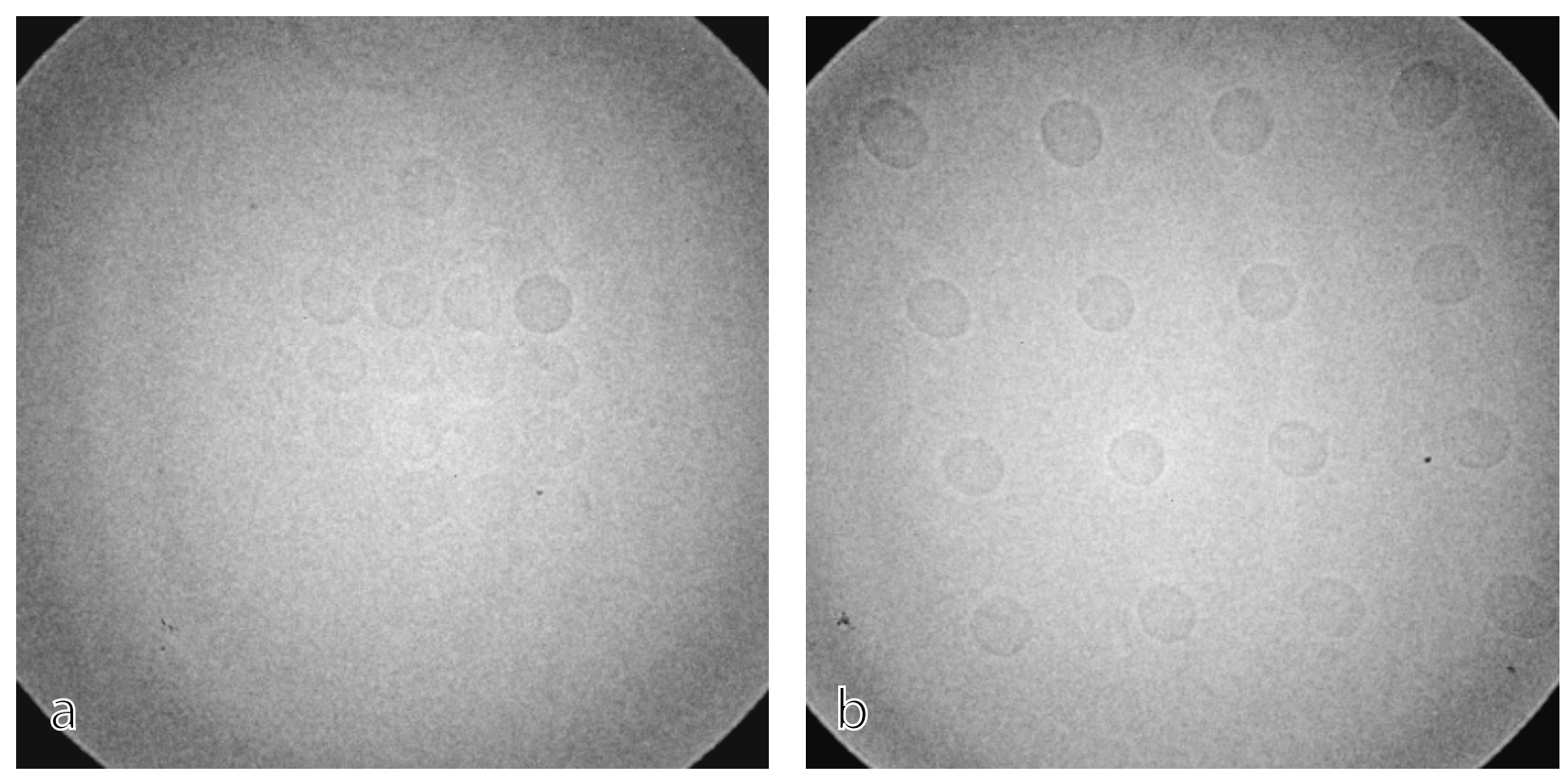

Downing et al., Figure 1 


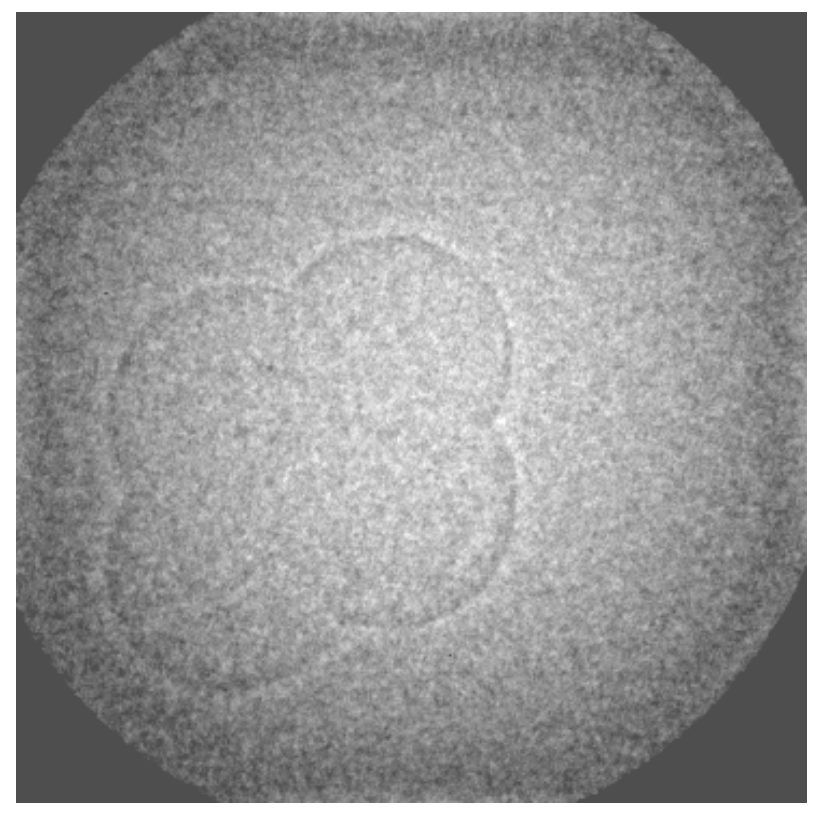

Downing et al., Figure 2 


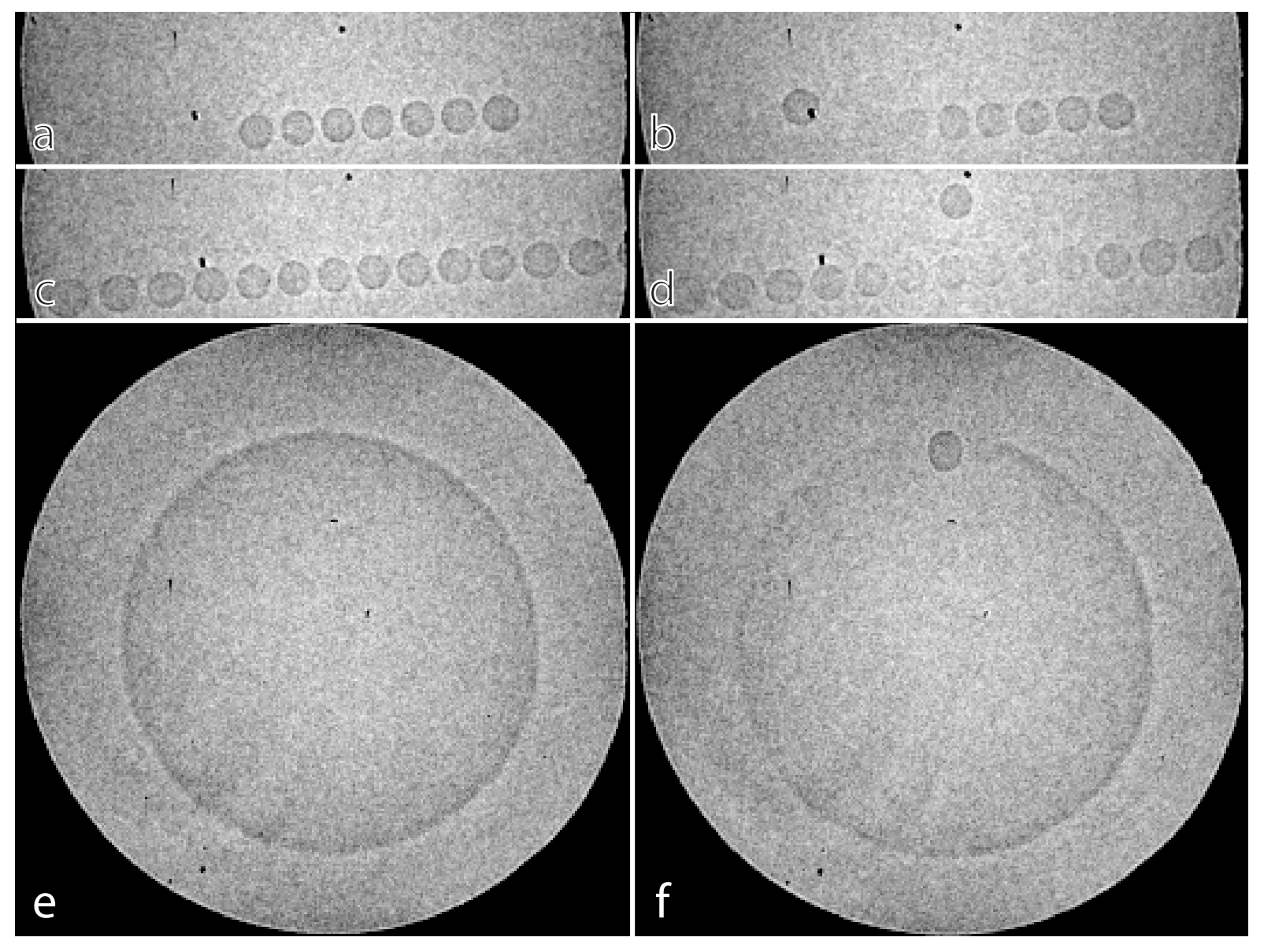

Downing et al., Figure 3 

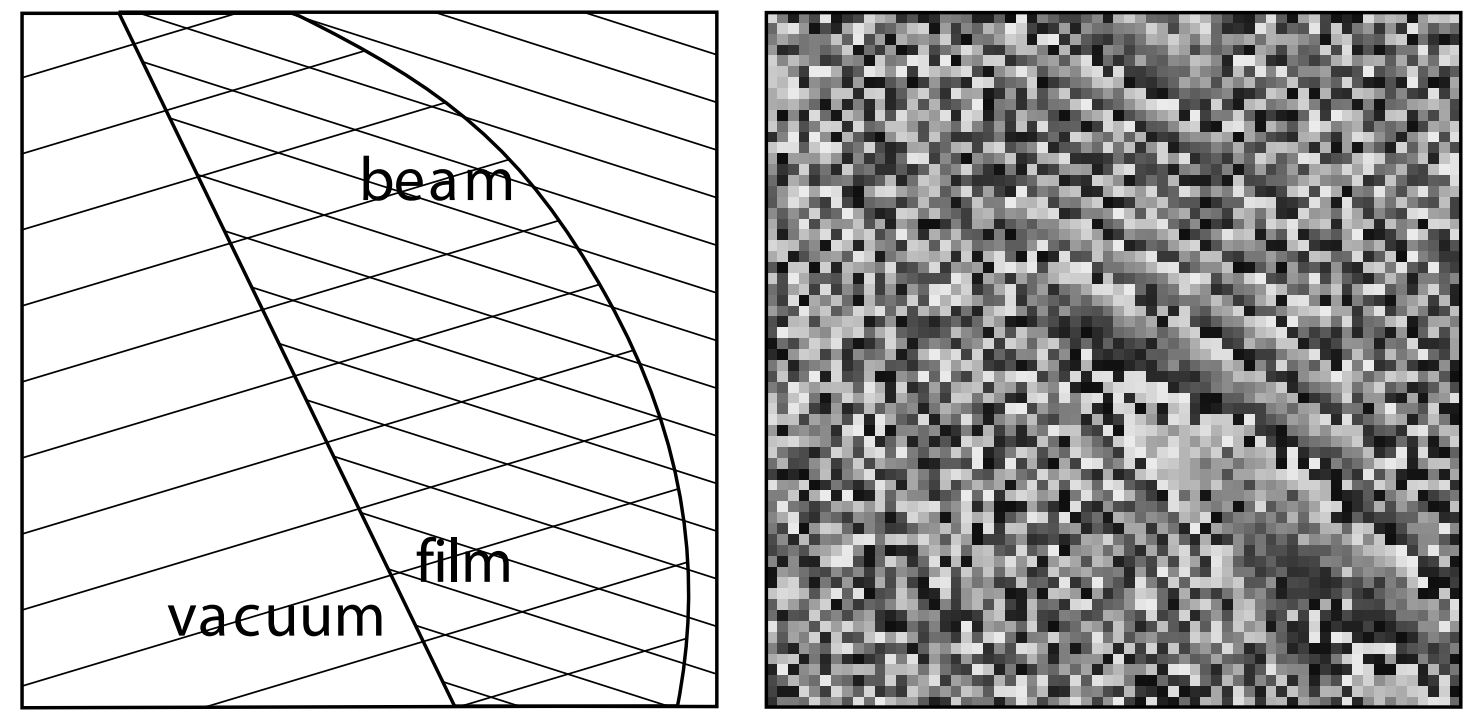

Downing et al., Figure 4 

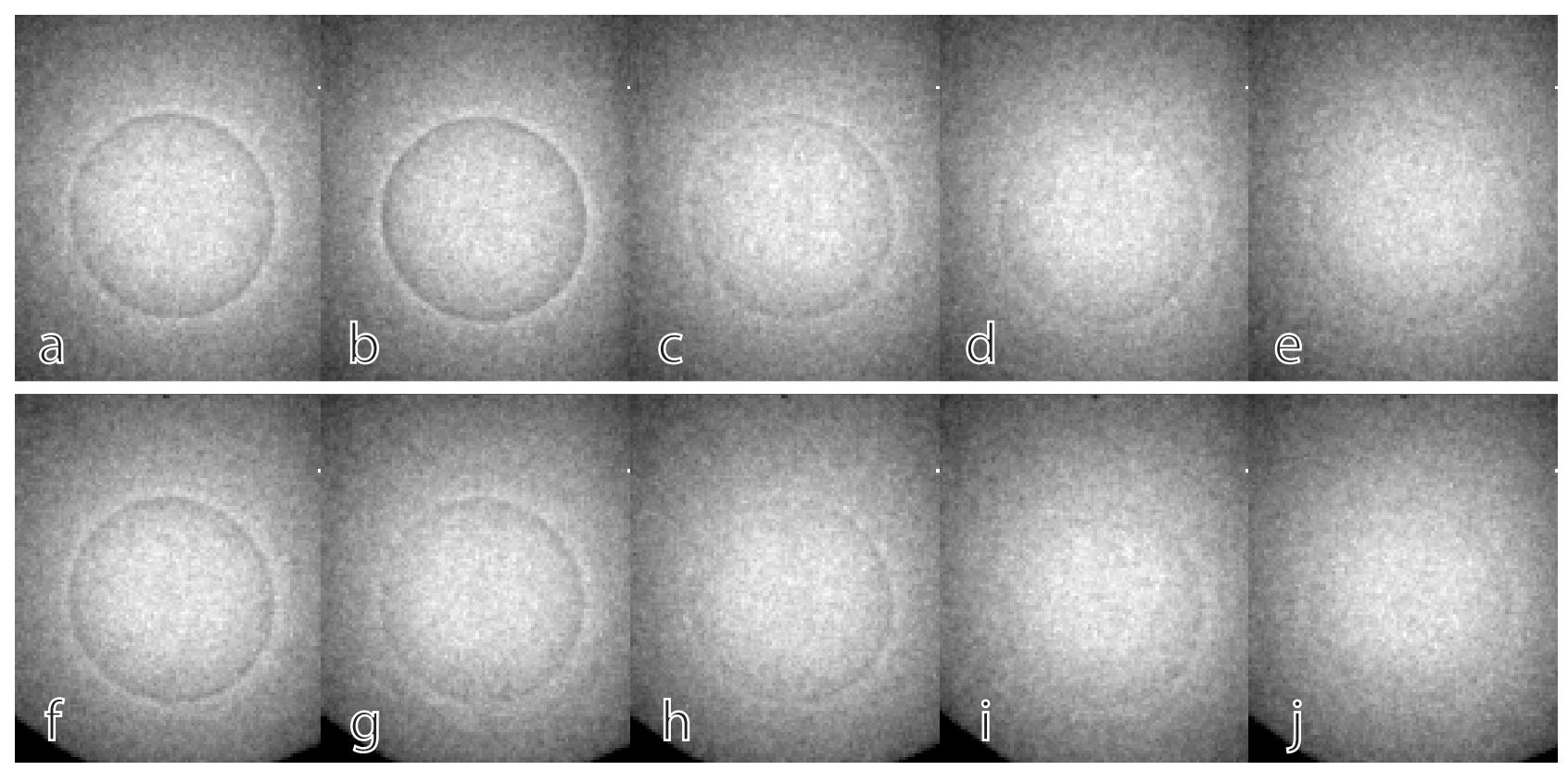

Downing et al., Figure 5 


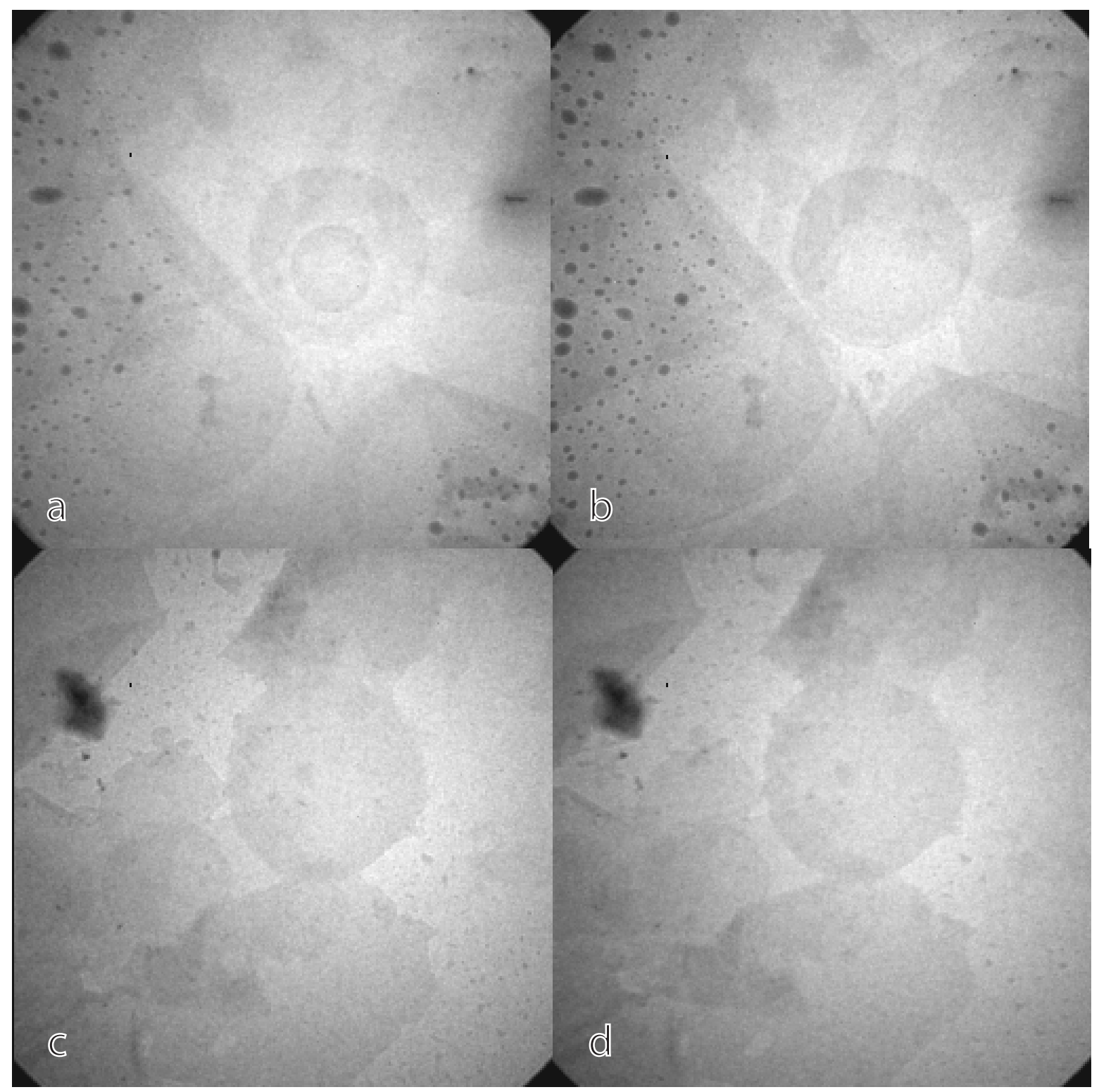

Downing et al., Figure 6 


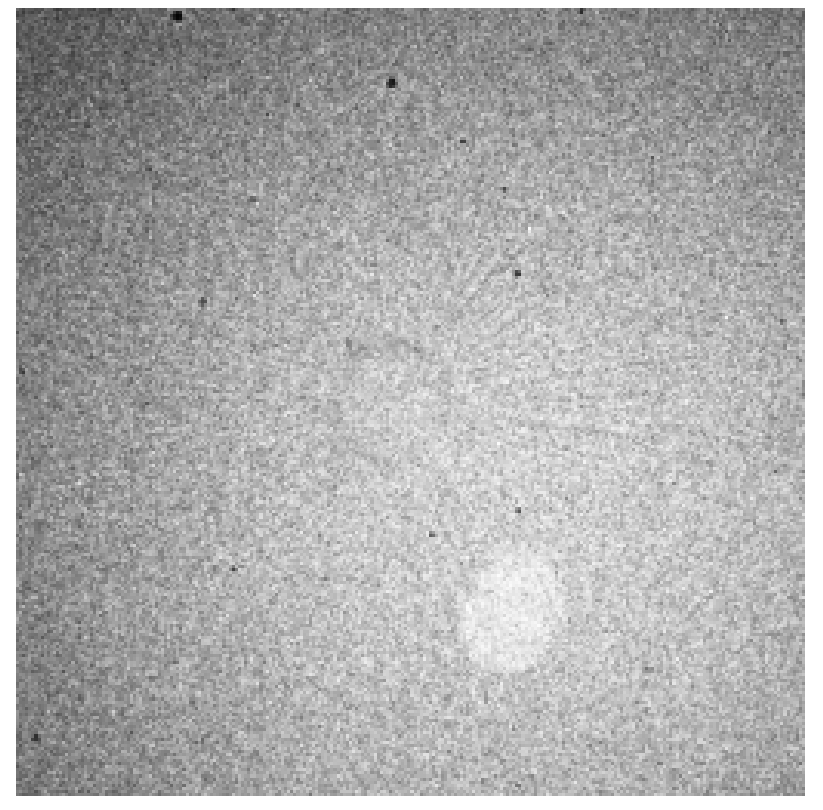

Downing et al., Figure 7 

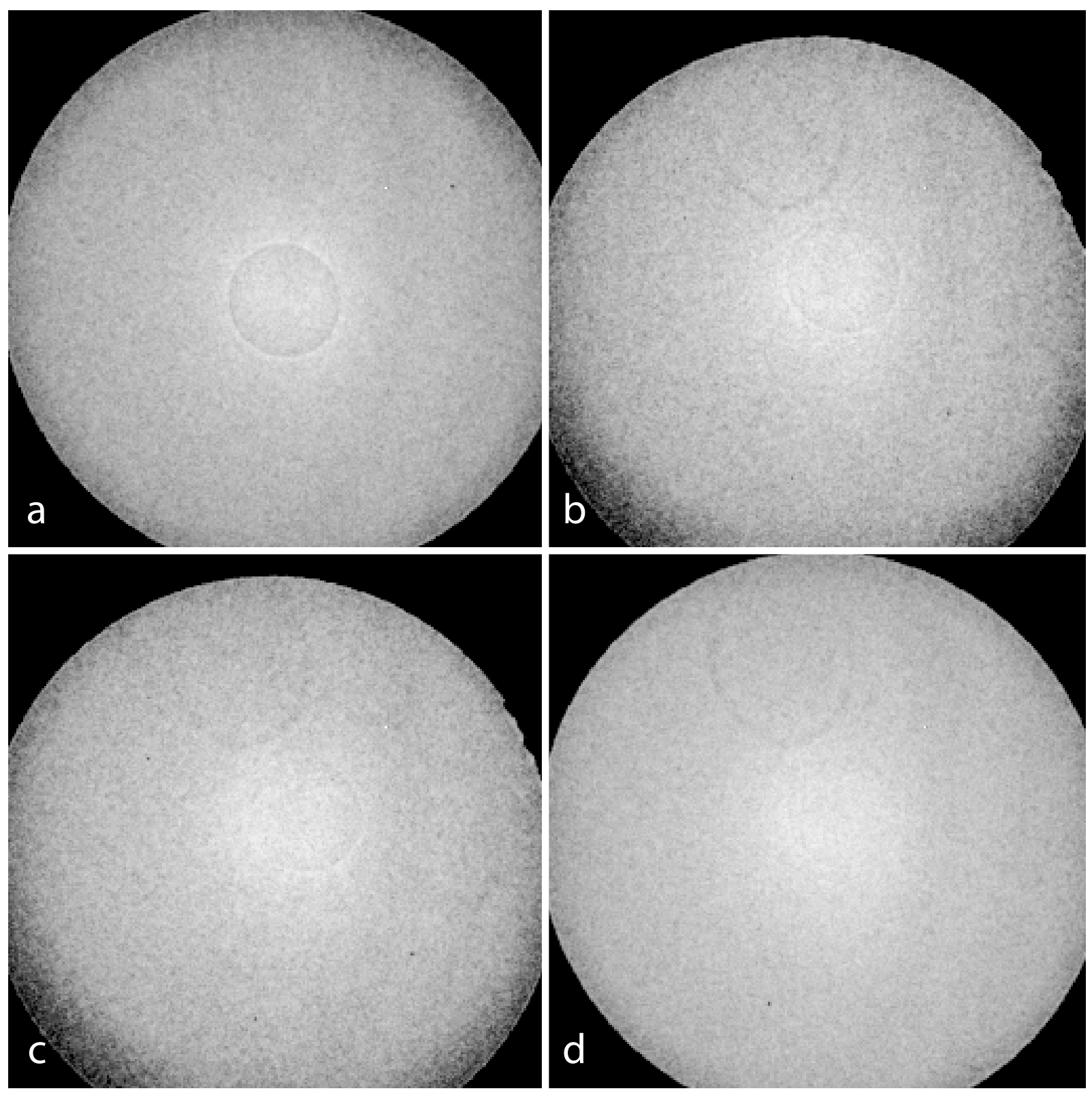

Downing et al., Figure 8 